\title{
Les Faux Amis Entre Les Langues Igbo Et Yoruba
}

\author{
Dr Kate Ndukauba \\ Abia State University, Uturu, Nigeria
}

\begin{abstract}
En traduction, les faux amis sont les mots qui, dans des langues différentes, semblent avoir le même sens mais qui en fait, ont des sens différents. Parfois, ils ont la même forme ou des formes identiques, ce qui peut dérouter un traducteur qui ne fait pas bien attention. Il peut y avoir des faux amis entre n'importe qu'elles langues en contact surtout pendant une activité traduisante. Dans cette communication, on compte relever les faux amis entre l'Igbo et le Yoruba, deux langues nigérianes qui appartiennent à la famille des langues dite Niger-Congo. On va parler des deux langues sous étude, discuter les causes des faux amis et les problèmes qui en découlent, et puis suggérer des moyens d'y faire face. Tout cela pour sensibiliser les apprenants et les professeurs des deux langues aussi bien que les traducteurs pour qu'ils fassent bien attention au cours de leur travail pour rester fidèle au sens de ce qui est dit, lu ou traduit.
\end{abstract}

Mots clés: traduction, langue, sens, fidélité, sémantique, orthographe.

\section{Introduction}

Les faux amis constituent un obstacle énorme à une bonne traduction, car le traducteur, s'il ne prend pas garde, peut facilement faire fausse route. Les faux amis se voient entre n'importe quelles langues, qu'elles soient internationales ou locales.

Dans cette présentation, on va relever quelques faux amis entre la langue Igbo et la langue Yoruba, deux langues locales nigérianes, dans l'objectif d'attirer l'attention des apprenants des langues et des traducteurs sur l'obstacle pour ne pas se faire piègés.

Ainsi va-t-on parler des faux amis en générale - ce que c'est, les causes et les problèmes qu'ils posent. Ensuite nous allons présenter brièvement les langues Igbo et Yoruba; et relever des faux amis entre elles. De là, nous ferons des observations dans l'intérêt des concernés.

\subsection{La langue Igbo}

La langue Igbo appartient à la branche Volta-Niger de la famille Niger-Congo. C'est la langue principale au Sud-est du Nigéria. Les igbophones nombrent de 18-25millions selon Omniglot, the language Encyclopedia.Wikipedia explique que la langue Igbo a plus de 20 dialectes y compris Enuani, Ngwa, Ohuhu, Onicha, Bonny. Opobo, Olu, Owere etc. Le système d'écriture, c'est l'alphabet Onwu. C'est une des langues majeures du Nigéria.

L'Igbo est une langue tonale avec deux tons distincts - haut et bas. Il y a parfois un troisième, ton haut minimisé ou dédramatisé. Des mots Igbo diffèrent par le ton. Par exemple: ákwá (pleurs), àkwà (lit), àkwá (oeuf), ákwà (pagne/tissue). Quoique les tons ne soient pas toujours écrits, ils essentiels pour la compréhension de ce qui est dit. Ils font sortir le sens de l'énoncé.

Il y a des variations de la langue Igbo à Cuba, au Trinidad et Tobago, à la République Dominicain, au Jamaïque, à Belize, à la Barbade et aux Bahamas.

\subsection{La langue Yoruba}

L'ethnie Yoruba nombre de 28millions d'après Wikipedia. La langue appartient, elle aussi, à la famille NigerCongo, comme l'Igbo. L'alphabet est du Latin. La langue Yoruba est apparentée à la langue Itsekiri du NigerDelta et Igala de la partie centrale du Nigéria. Le Yoruba est la langue nigériane la plus parlée hors de l'Afrique. Omniglot dit qu'on parle cette langue au Sud-Est du Nigéria, au Bénin, au Togo, au Royaume-Uni, au Brésil, aux Etats-Unis et une variété de Yoruba aux Caraïbes.

Il y a plusieurs dialectes de Yoruba, réunis en trois groupes:

Le Yoruba du Nord-Est parlé à Abeokuta, Ibadan, Oyo, Ogun et Lagos. 
Le Yoruba Central parlé à Igbomina, Yagba, Ilesa, Ife, Ekiti, Akure, Efon et Ijebu.

Le Yoruba du Sud-Est parlé à Okitipupa, Ilaje, Ondo, Owo, Ikare, Sagamu, et des parties d'Ijebu.

La langue Yoruba a des signes diacritiques. Elle a trois tons - haut, bas et mi (central). Chaque syllable a, au moins, un ton. Le mi-ton n'a pas de signe diacritique.

\subsection{Les faux amis}

Au sens littéral, les faux amis sont les amis qui ne le sont pas en réalité, mais qui paraissent l'être. Dans le domaine lingustique, il s'agit des mots ou expressions des langues différentes qui paraissent dire la même chose alors qu'ils ne le disent pas. Des experts ont défini les faux amis. Le Dictionary of Translation Studies les définissent en ces termes:

"a standard term used to describe source- language and target-language items which have the same or very similar form but different meanings, and which consequently give rise to difficulties in translation (and indeed interlingual communication in general)"

"un terme normal pour décrire des termes de langue-source et langue-cible, qui ont la même forme ou formes identiques mais des sens différents et par conséquent, donne naissance aux difficultés en traduction (surtout en communication interlinguale en générale). (Notre traduction)

Pour Ojo, c'est "un phénomène dans lequel des mots ayant des orthographes identiques ou presque identiques dans deux langues mais avec des sens complètement différents. Il ajoute que les faux amis présentent des alternatifs attirants à l'apprenant ou à l'usager des deux langues. (13)

Ajunwa explique ainsi,

"In translation theory, the expression is used to describe words even structures, which may appear to correspond semantically in both the source and target languages by virtue of their etymology and form, but actually do not, due to their separate evolutions in each of the languages". (167)

“Dans la théorie de traduction, l'expression "faux amis' décrit des mots ou même des structures qui semblent correspondre au niveau sémantique dans la langue-source et la langue-cible en raison de leur étymologie et forme, mais qui en réalité, ne l'est pas à cause de l'évolution distincte dans chacune des langues". (Notre traduction)

D'après Dictionary of Language Teaching and Applied Linguistics, les faux amis sont les mots qui ont la même forme ou presque mais des sens différents dans les deux langues.

Alors, les faux amis se ressemblent en forme, ce qui fait que l'apprenant d'une des langues leur attribue le même sens alors qu'ils ne veulent pas dire la même chose.

Les faux amis se manifestent souvent entre deux langues qui appartiennent à la même famille, mais on s'en aperçoit aussi entre des langues non-apparentées. Ils proviennent de l'interférence linguistique entre deux langues en contact surtout quand le sens de deux mots sont proches l'un de l'autre. La difference entre les mots ou les expressions peut être au niveau de la connotation ou de la forme.

Wandruszka (1978:228) cité par Shuttleworth est d'avis que si un 'faux ami d'une langue-cible est fréquemment employé dans le sens de la langue-source, il peut se transformer en un 'vrai ami'en se revêtissant du sens de la langue-source tout en retenant son propre sens. (1978:228)

Les faux amis sont des exemples frappants de l'interférence linguistique. Ils sont évidents au niveau de l'orthographe, du sens et même de l'intonation dans le cas des langues tonales, surtout les langues africaines.

Un étudiant igbo qui apprend le Yoruba fait toujours face à ce problème de l'interférence linguistique parce que beaucoup de mots Yoruba ressemblent aux mots igbo et il y a la tentation de les employer dans le même sens, ce qui peut aboutir aux faux amis. La similarité des mots ou des expressions fait que l'apprenant d'une deuxième langue, emploie les mots dans un faux sens.

\subsection{Les causes des faux amis}

Wikipedia énumère quelques causes des faux amis 
1. Une étymology partagée: Des fois, langue A emprunte un mot de langue B ou tous les deux empruntent de langue $\mathrm{C}$ ou héritent le mot d'un ancêtre commun.Plus tard, il y a une mutation de sens ou le mot acquiert un sens supplémentaire dans une des langues. Par exemple:

preservative (anglais) - qui garde, sauve ou garantit contre

préservatif(français) - condom

2. Les homonymes: Ce sont des mots de prononciation identique et de sens différents. Les faux amis évoluent séparemment dans des langues différentes. Des mots peuvent changer de prononciation au cours des années et parfois converger sur la même prononciation ou apparence quoiqu'ils viennent des origines différentes.

3. Les pseudo-anglicismes: Il s'agit ici des nouveaux mots qui viennent des morphèmes anglaises indépendamment des constructions anglaises comparatives et avec un sens différent intentionnel.

Exemple: oldtimer - an old car

handy - a mobile phone

beamer - a computer projector or video projector

Il ajoute que le français et l'allemand ont beaucoup de pseudo-anglicismes qui proviennent du gérondif anglais.

Exemple: $\quad$ parking (allemande) - the act of parking a car

parking (français) - parking lot.

4. L'emprunt des mots d'une autre langue peut aboutir aux faux amis. Par exemple:

gymnasium (latin) - un lieu d'éducation (allemand)

- un lieu d'exercice physique (anglais)

\subsection{Les problèmes des faux amis}

Sans doute les faux amis posent-ils des problèmes surtout aux apprenants des langues et aux traducteurs. Ces problèmes comptent les suivants:

1. Des mots similaires peuvent ne pas comprendre toutes les nuances du mot dans les deux langues. Par exemple: 'demande' en anglais ne veut pas dire exactement la même chose en français.

2. Un chevauchement partiel de sens crée des complications supplémentaires

3. Les faux amis font que les apprenants identifient mal les mots à cause de l'interférence linguistique.

4. Les faux amis créent également des difficultés entre ceux qui parlent les dialectes de la même langue, comme une expression peut se revêtir de sens différent dans des dialectes différents.

5. Un cas problématique des faux amis se produit quand un des deux mots est révoltant ou dénigrant.

Exemple: $\quad$ spaz - une personne maladroite (clumsy person) - anglais américain

$$
\text { - un handicappé (a disabled person) - anglais britannique }
$$

\subsection{Les faux amis Igbo-Yoruba}

\begin{tabular}{|l|l|l|}
\hline & IGBO & YORUBA \\
\hline 1. & Abọ (une corbeille) & Abọ (retour) \\
\hline 2. & Ada (la première fille) & Ada (le couteau) \\
\hline 3. & Ala (terre) & Ala (le rêve) \\
\hline 4. & Anya (oeil) & Aya (le coeur) \\
\hline 5. & Anụ (la viande) & Anụ (compassion) \\
\hline 6. & Ara (seins) & Ara (corps) \\
\hline 7. & Awo (crapaud) & Awọn (ils, eux) \\
\hline 8. & Dede (frère aîné) & Dede (également) \\
\hline 9. & Ede (tarot) & Ede (langue) \\
\hline 10. & Eji (escargot) & Eji (deux) \\
\hline 11. & Enyi (ami) & Eỵ̣n (vous) \\
\hline 12. & Enyi (ami) & Eyi (ceci) \\
\hline 13. & Ewu (chèvre) & Ewu (vêtement) \\
\hline & & \\
\hline
\end{tabular}




\begin{tabular}{|c|c|c|}
\hline 14. & Ibe ji (un morceau d'igname) & Ibeji (jumeau/jumelle) \\
\hline 15. & Ji (igname) & Ji (réveille) \\
\hline 16. & Ji oko (une espèce d'igname) & Joko (assieds-toi) \\
\hline 17. & Ibi (hernie) & Ibi (ici) \\
\hline 18. & Ife (fable) & Ifẹ (amour) \\
\hline 19. & Efe (occasion), efe (vêtement) & Efọ (légume) \\
\hline 20. & Igba (tam-tam) & Igba (temps) \\
\hline 21. & Igbe (boîte) & Igbe (fèces) \\
\hline 22. & Igbo (tribu) & Igbo (brousse) \\
\hline 23. & Igbo (héroïne) & Igbo (brousse) \\
\hline 24. & Ile (une espèce de poisson) & Ile (maison) \\
\hline 25. & Ilu (amer) & Ilu (ville) \\
\hline 26. & Imeji (foie) & Meji (deux) \\
\hline 27. & Imo (nom de rivière) & Imọ (sagesse) \\
\hline 28. & Inu (entendre) & Inu (inside) \\
\hline 29. & Iwe (colère) & Iwe (livre) \\
\hline 30. & Iya (réponse à une salutation) & Iya (mère), iya (punition) \\
\hline 31. & Miiri (soupe en dialecte ngwa) & Miiri (je ne le vois pas) \\
\hline 32. & Odo (cadeau) & Odo (ruisseau) \\
\hline 33. & Odo (sentier), odo (pilon) & Odo (zéro) \\
\hline 34. & Ofe (soupe) & Ọfẹ (gratuit) \\
\hline 35. & Ogbonna (nom propre) & O gbona ( c'est chaud) \\
\hline 36. & Ogo (hauteur) & Ogo (gloire) \\
\hline 37. & Oja (flûte) & Oja (marché), o ja (il est casse) \\
\hline 38. & Oji (kola), oji (noir) & Oji (il se réveille) \\
\hline 39. & Oke (part), & Oke (haut) \\
\hline 40. & Oke (mâle) & O ke (il a pleuré) \\
\hline 41. & Ola (anneau) & Ola (demain) \\
\hline 42. & Oluku (imbécile) & Olukọ (professeur) \\
\hline 43. & Ona (une espèce d'igname) & Ọna (route) \\
\hline 44. & Ori (beurre de karité) & Ori (tête) \\
\hline 45. & Oro (jeu d'enfant) & Orọ (parole) \\
\hline 46. & Ose (poivre) & Ose (merci) \\
\hline 47. & Ose (dé) & O se (il a fait) \\
\hline 48. & Otuto, otito (louanges) & Otitọ (vérité) \\
\hline 49. & Tutu (avant), & Tutu (froid) \\
\hline 50. & Ekpo (mascarade) & Epo (huile) \\
\hline 51. & Bata (entre!) & Bata (chaussure) \\
\hline 52. & Obe (croix) & Ọbẹ (soupe) \\
\hline
\end{tabular}

\section{Observations/Conclusion}

Des plusieurs cas de faux amis relevés entre l'Igbo et le Yoruba, on constate des ressemblances dans l'orthographe, la prononciation et les signes diacritiques. Les deux langues appartiennent à la même famille donc, ells sont apparentées et on doit y avoir des faux amis. On note ici, le rôle essentiel que jouent les tons et les signes diacritiques. Qu'ils soient au-dessus ou au-dessous des lettres, ces signes modifient l'orthographe, la prononciation et le sens des mots. Etant des langues tonales, il faut toujours mettre les signes pour render la compréhension facile.

$$
\begin{aligned}
& \text { Exemple: odo-ruisseau, odo - zero } \\
& \text { iya-mère, iya-punition }
\end{aligned}
$$

C'est vrai que les faux amis créent la confusion chez un apprenant d'une deuxième langue ou un traducteur qui ne fait pas bien attention et par consequent, les mots sont employés incorrectement. Le plus intéressant, c'est que malgré les problêmes posés par les faux amis, il y a toujours un côté positif. On est d'accord avec Ajunwa que les faux amis permettent une fécondation croisée des langues. Il y a l'enrichissement syntactique et néologique de plusieurs langues parce que ces faux amis parfois finissent par devenir des vrais amis. (11) 
Les processus d'emprunt, de calque, de création des mots, de dérivation, de composition, tous aident les langues à acquérir de nouveau vocabulaire fonctionnel. Un mot arrive donc à avoir un nouveau sens qui ne se trouvait pas dans la langue originale.

Dans des situations linguistiques, des faux amis résultent en changement sémantique, c'est-à-dire, la naissance d'un vrai nouveau sens qui est alors employé fréquemment dans une langue.

De tout ce qui précède, on voit ce que c'est que les faux amis, avec ses causes, ses problèmes et ses effets. On a établi que les faux amis existent entre toutes les langues - locales, nationales ou internationales, y compris les dialectes d'une même langue. Donc, il faut toujours prendre garde quand on travaille avec des langues différentes.

\section{Références}

[1] E.Ajunwa, A Textbook of Translation Theory and Practice. Awka: ENOVIC Ltd, 2014.

[2] H. Bertaud du Chazaud, Dictionnaire des Synonymes. Paris: Dictionnaire Le Robert, 1989.

[3] Dictionary of Translation Studies. Uk: St Jerome Publishing, 1997.

[4] A. S. Ojo, A Comprehensive Revision Handbook of French Grammar. Ibadan: Signal Education Services Ltd, 2002.

[5] J. Richards and R. Schmidt Dictionary of Language Teaching and Applied Linguistics. 3e ed. England: Pearson Education Ltd, 2012.

[6] Wikipedia, the free Encyclopedia. "Igbo language" http://en.wikipedia.org/igbo_language. October 26, 2014

[7] Wikipedia, the free Encyclopedia. "False friends" http://en.wikipedia.org/false_friends October 26, 2014

[8] Wikipedia, the free Encyclopedia. "Yoruba language" http://en.wikipedia.org/yoruba language October 26, 2014

[9] Wikitravel.org/en/igbo_phrase October 26, 2014

[10] www.omniglot.com/writing/igbo.htm October 26, 2014

[11] www.omniglot.com/writing/yoruba.htm October 26, 2014 